\title{
Coincidence of aortic valve stenosis and regurgitation and multiple cardiac papillary fibroelastomas in a young male adult
}

\author{
Karsten Sydow, MD, ${ }^{\text {a }}$ Sonja Schrepfer, MD, ${ }^{\text {b }}$ Olaf Franzen, MD, ${ }^{\text {a }}$ Christian Detter, MD, ${ }^{\text {b }}$ Stephan Willems, MD, \\ Thomas Meinertz, MD, and Hermann Reichenspurner, MD, PhD, ${ }^{\mathrm{b}}$ Hamburg, Germany
}

Primary tumors of the heart are rare. ${ }^{1,2}$ CPFs, the second most common primary cardiac tumors, are benign endocardial papillomas predominantly affecting the cardiac valves. ${ }^{3}$ Although CPFs occur in all age groups, ranging from the neonatal period to the 10th decade of life, they clearly predominate in adults and are particularly frequent between the fourth and eighth decades of life. CPFs are more common in male subjects in most case series. They are generally slow-growing tumors but might serve as a nidus, allowing formation of large superimposed thrombi over a short period of time and therefore might result in life-threatening complications. ${ }^{4}$ Although the CPF is a morphologically distinctive cardiac lesion, its histogenesis remains controversial.

\section{Clinical Summary}

A 35-year-old man was admitted with acute pulmonary edema. He had a 3-month history of progressive dyspnea under ordinary exertion accompanied by chest pain. The patient was severely ill at presentation, with orthopnea and cold sweats. Respirations were 27 breaths/min, and fine inspiratory crackles were audible across the whole chest. The patient had sinus tachycardia, and a previously unknown $4 / 6$ systolic murmur was audible in the aortic position. This murmur was transmitted into the carotid arteries and was accompanied by a diastolic murmur and a muted second heart sound. Hematologic, coagulatory, and fibrinolytic tests showed no abnormalities. The C-reactive protein level $(1.8 \mathrm{mg} / \mathrm{dL})$ was slightly above normal range.

Transthoracic and transesophageal echocardiography revealed a slightly impaired left ventricular function. The aortic valve showed pronounced echocardiography-dense thickened leaflets, merging into the left ventricular outflow tract (LVOT) and toward the basis of the anterior mitral leaflet (Figure 1). Morphologically, almost no opening movement was detectable, and the assessed aortic valve opening area was $0.75 \mathrm{~cm}^{2}$, with a gradient of $110 / 70$ $\mathrm{mm} \mathrm{Hg}$ and a severe aortic valve regurgitation. In transition of the anterior left ventricular wall to the LVOT, a $3 \times 3-\mathrm{mm}$ mobile structure was evident (Figure 2, A). In the immediate vicinity an

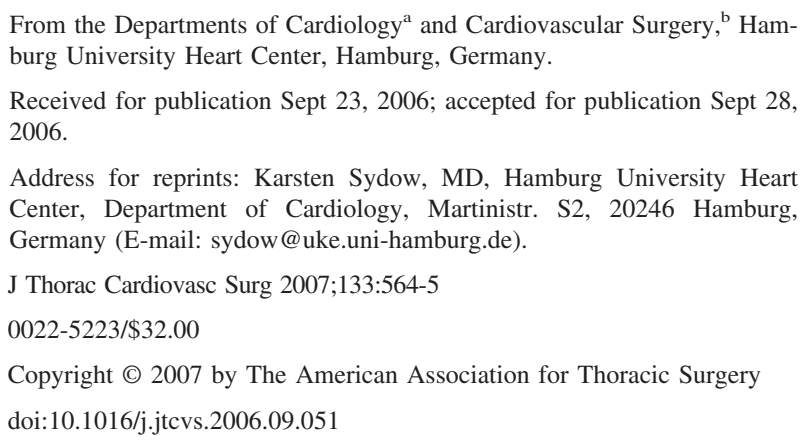

additional mobile structure was present on a thickened chorda tendinea, ranging from the anterolateral papillary muscle to the central and anterolateral anterior mitral leaflet. In the middle of both papillary muscles, aberrant chordae tendinea was present between the 2 muscles, with another mobile structure between the anterolateral papillary muscle and the anterolateral ventricular wall (Figure 2, B).

Cardiac surgery revealed a morphologically inconspicuous mitral valve. Inspection of the subvalvular region and the left ventricle revealed multiple gelatin-like tumors distributed all over the heart, including the chordae tendineae. The aortic valve leaflets were impossible to differentiate, resulting in an almost monocuspid aortic valve. Resection of the aortic valve was complicated by an excessive calcification, which included the LVOT and the intraventricular septum. Multiple gelatin-like tumors were spotted toward the aortic valve in the LVOT and the mitral valve apparatus. A curative excision of the tumor appeared to be impossible because of its extensive spreading. Therefore 2 masses were resected from the LVOT and the anterior papillary muscle/chordae tendineae for histologic examination. Microscopy revealed a papillary tumor with a central core of collagen and elastic fibers beside small-lesion calcifications and villus fronds covered by an endothelial layer without any cellular atypia found within the mass, which is consistent with the diagnosis of cardiac papillary fibroelastoma (CPF). Histologic examination of the excised aortic valve revealed a degenerative sclerosis of the valve consistent with a prior endocarditis.

The patient had an uneventful postoperative course and was discharged after adequate oral anticoagulation with phenprocoumon. One-year follow-up transesophageal echocardiography showed good

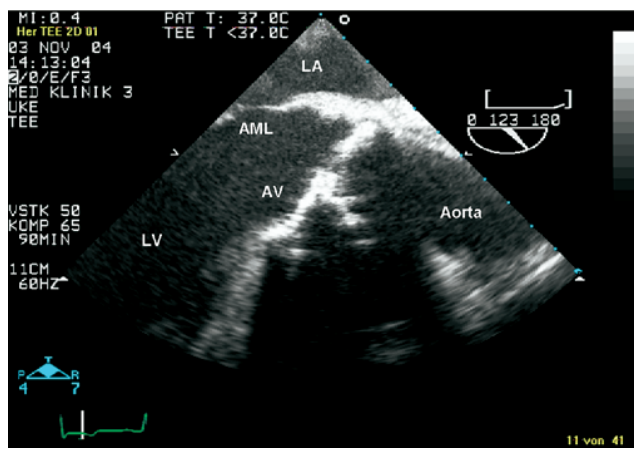

Figure 1. Transesophageal 3-chamber view. $L A$, Left atrium; $A M L$, anterior mitral leaflet; $A V$, aortic valve; $\boldsymbol{L V}$, left ventricle. 

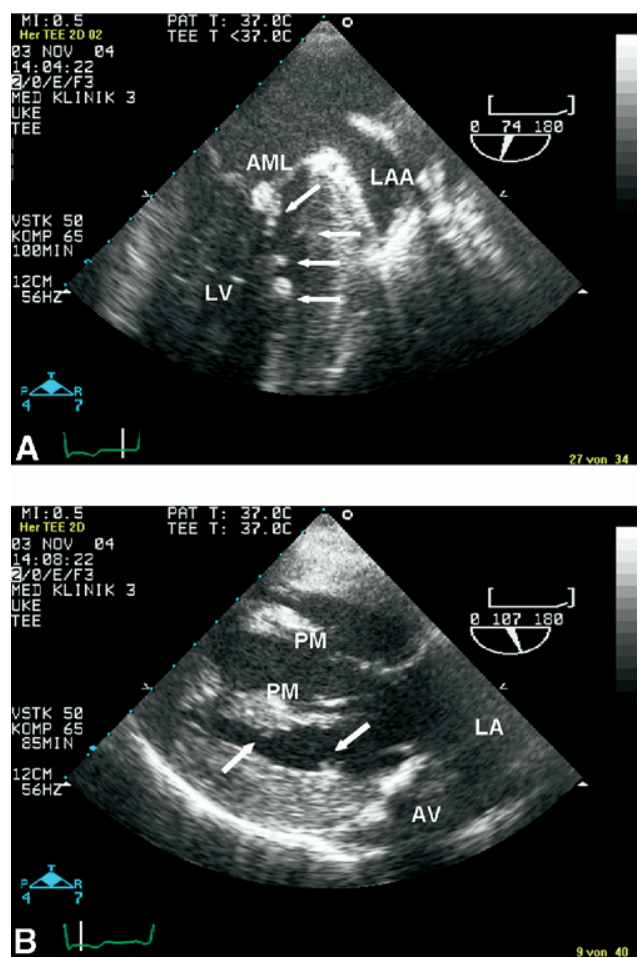

Figure 2. A, Transesophageal 2-chamber view. AML, Anterior mitral leaflet; $L A A$, left atrial appendage; $L V$, left ventricle. $B$, Transesophageal-transgastric long-axis view. PM, Papillary muscle; $L A$, left atrium; $A V$, aortic valve. function of the aortic valve and no further growth or additional tumor growth after cardiac surgery.

\section{Discussion}

This case describes a patient with the unusual finding of multiple cardiac fibroelastomas involving the mitral valve and the LVOT in addition to an altered aortic valve after prior valve endocarditis. We hypothesize that the local accumulation of mobile structures might be, at least in part, because of the congenital altered bicuspid aortic valve and consecutive inflammatory alteration. Furthermore, accelerated by aortic regurgitations, jet lesions in the subvalvular region of the mitral valve and the adjacent left ventricle might have contributed to the development of the tumor. After surgical replacement of the formerly infected aortic valve, tumor growth appeared to discontinue.

\section{References}

1. Reynen K. Frequency of primary tumors of the heart. Am J Cardiol. 1996;77:107.

2. Goldberg HP, Glenn F, Dotter CT, Steinberg I. Myxoma of the left atrium; diagnosis made during life with operative and post-mortem findings. Circulation. 1952;6:762-7.

3. Virmani R, Burke A, Farb A, Atkinson JB. Cardiovascular pathology. Major problems in pathology. Vol. 40. Philadelphia: WB Saunders; 2001.

4. Joffe II, Jacobs LE, Owen AN, Ioli A, Kotler MN. Rapid development of a papillary fibroelastoma with associated thrombus: the role of transthoracic and transesophageal echocardiography. Echocardiography. 1997; 14:287-92.

\section{Facile conversion from mechanical to bioprosthetic composite aortic root replacement}

Luca A. Vricella, MD, and Duke E. Cameron, MD, Baltimore, Md

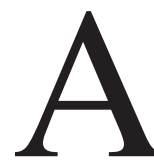

ortic root replacement with a composite valve graft mechanical prosthesis has become established therapy for acute and chronic pathology that eventually leads to enlargement, dissection, or rupture of the aortic root. Although minimal early and late morbidity and mortality have been

From the Division of Cardiac Surgery, The Johns Hopkins Medical Institutions, Baltimore, Md.

Received for publication Oct 12, 2006; accepted for publication Oct 24, 2006.

Address for reprints: Luca A. Vricella, MD, FACS, Division of Cardiac Surgery, The Johns Hopkins Hospital, 600 N Wolfe St, Blalock 618, Baltimore, MD 21287 (E-mail: 1vricella@jhmi.edu).

J Thorac Cardiovasc Surg 2007;133:565-7

$0022-5223 / \$ 32.00$

Copyright () 2007 by The American Association for Thoracic Surgery doi:10.1016/j.jtcvs.2006.10.024 reported, some patients will require operative reintervention on the neoaortic root, with the

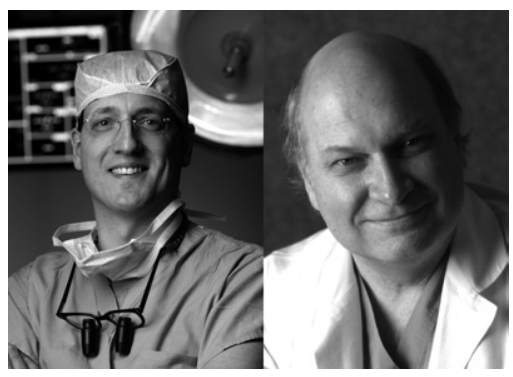

Drs Vricella and Cameron (left to right) goal of conversion from a mechanical to a xenograft aortic valve prosthesis.

We describe an easily reproducible technique that aims at minimizing the degree of complexity of such reintervention while still achieving the goal of freedom from anticoagulation.

\section{Clinical Summary}

A 38-year-old man with a history of Marfan syndrome and intravenous drug abuse presented to our institution in 1999 with symptomatic aneurysmal dilation of the aortic root and aortic regurgitation and underwent aortic root replacement with a 27-mm composite mechanical valved prosthesis (St Jude Medical, St Paul, Minn). Despite enrollment in a drug rehabilitation program, the 